\title{
Relación entre estilo de vida y control metabólico en pacientes con Diabetes Mellitus Tipo 2 de Chota, Perú
}

\begin{abstract}
Relationship between life style and metabolic control among patients with type 2 diabetes in Chota, Peru
\end{abstract}
José Ander Asenjo-Alarcón ${ }^{1, a, b}$

\section{RESUMEN}

Objetivo: Determinar la relación del estilo de vida con el control metabólico en pacientes con diabetes mellitus tipo 2, de Chota, Cajamarca. Material y métodos: Estudio observacional, transversal, en 102 pacientes identificados en el Centro Médico de EsSalud. Los estilos de vida se determinaron con el cuestionario IMEVID y el control metabólico con una guía de interpretación diagnóstica adaptada de la Asociación Americana de Diabetes (ADA). Se determinaron frecuencias absolutas y relativas, el chi cuadrado de independencia y coeficiente gamma con un nivel de confianza del 95\%. Resultados. Los pacientes presentaron un estilo de vida desfavorable (45,1\%), sobrepeso (43,1\%), perímetro abdominal elevado (53,9\%), hiperglucemia $(62,7 \%)$, C-LDL elevado (79,4\%), C-HDL disminuido (59,8\%), triglicéridos elevados $(61,8 \%)$ y mal control metabólico de la enfermedad (61,8\%). Conclusiones: Los pacientes en su mayoría tenían un estilo de vida desfavorable, existe una relación directa y altamente significativa entre el estilo de vida y el control metabólico de los pacientes; el estilo de vida desfavorable, se asoció a mal control metabólico de la enfermedad.

PALABRAS CLAVE: Estilo de vida, diabetes mellitus tipo 2, síndrome metabólico. (Fuente: DeCS BIREME).

\section{SUMMARY}

Objective: To determine the relationship between life style and metabolic control among patients with type 2 diabetes in Chota, Cajamarca. Methods: cross sectional study among 102 patients at Centro Médico de EsSalud. Life style was determined using the IMEVED questioner, and metabolic control was evaluated with a guideline adapted from the American Diabetes Association (ADA). Relative and absolute frequencies were calculated, chi square and the gamma coefficient with a $95 \%$ level of confidence were used. Results: Unfavorable life style was observed in $45.1 \%$, overweight in $43.1 \%$, increased abdominal perimeter in $53.9 \%$, hyperglycemia in $62.7 \%$, high C-LDL in $79.4 \%$, reduced C-HDL in $59.8 \%$, high triglycerides in $61.8 \%$ and bad metabolic control in $61.8 \%$. Conclusions: Most patients had an unfavorable life style, a direct relationship between life style and metabolic control was found. An unfavorable life style was associated with poor metabolic control.

KEYWORDS: Life style, type 2 diabetes mellitus, metabolic syndrome. (Source: MeSH NLM). 


\section{INTRODUCCIÓN}

La diabetes mellitus tipo 2(DM2) es una enfermedad crónica no transmisible que evoluciona con una serie de complicaciones y limitaciones graduales, por ello es fundamental que surjan modificaciones paulatinas en el estilo de vida de los pacientes para mantener un control metabólico adecuado (1). No obstante, gran parte de los pacientes son indiferentes a los cambios que tienen que asumir. Indudablemente, esta patología constituye un problema de salud pública, que ocasiona un alto impacto económico y social y disminución en la calidad de vida de los sujetos que la sufren, así como la pérdida de años de vida productiva y años de vida potencial, a consecuencia de las complicaciones crónicas, desórdenes en el estilo de vida o de la mortalidad (2).

En el Perú, la prevalencia de DM2 es de 7\%, según regiones geográficas, en la costa el $8,2 \%$ de la población la padece, $4,5 \%$ en la sierra y $3,5 \%$ en la selva. A nivel de Lima es de 8,4\% (3).

El estilo de vida inadecuado ha traído como consecuencia que las cifras de DM2 sigan aumentando a nivel mundial, principalmente el consumo excesivo de calorías, manifestado por sobrepeso (39\%), obesidad (13\%) y la inactividad física (4); en el Perú el porcentaje es aún mayor, la población adulta presenta $42,5 \%$ de sobrepeso y $19,8 \%$ de obesidad (5).

Existen evidencias de que una gran proporción de casos de DM2 y sus complicaciones pueden prevenirse con una dieta saludable, actividad física regular, mantenimiento de un peso corporal normal y evitar el consumo de tabaco y alcohol (6).

En la Región Cajamarca y particularmente en Chota, el uso de la tecnología y los cambios introducidos por la sociedad actual han provocado que las personas modifiquen sus estilos de vida en cuanto a alimentación, trabajo y uso del tiempo libre, haciéndolas menos saludables; sumado a esto el incremento paulatino de DM2 y la existencia de escasos estudios en el contexto descrito, justifica el desarrollo de la investigación.

El estudio tuvo como objetivo determinar la relación entre el estilo de vida y el control metabólico en pacientes con DM2 de Chota, Cajamarca.

\section{MATERIAL Y MÉTODOS}

Estudio observacional, transversal, realizado durante los meses de febrero a marzo de 2015.

La población estudiada fue de 102 pacientes con DM2. Se incluyeron pacientes de ambos sexos entre 30 y 87 años de edad, registrados y atendidos en el Servicio de Programas del Centro Médico EsSalud de Chota hasta el 31 de diciembre del 2014, que contaban con historia clínica completa en la Oficina de Admisión. Se excluyeron a los pacientes que no asistían periódicamente a sus controles.

Las variables principales del estudio fueron:

Estilo de vida: Se determinó de acuerdo al cuestionario Instrumento de Medición de Estilo de Vida en pacientes con DM2 (IMEVID), estilo de vida desfavorable ( $<60$ puntos), poco favorable (60 a 80 puntos) y favorable ( $>80$ puntos) (7).

Control metabólico: Ésta se clasificó en: Buen y mal control metabólico. El buen control metabólico se estableció cuando las variables evaluadas se encontraban dentro de los valores normales. Se consideraron las siguientes variables: IMC (normal: < $25 \mathrm{Kg} / \mathrm{m}^{2}$, sobrepeso: $25-29,9 \mathrm{Kg} / \mathrm{m}^{2}$ y obesidad: $\geq 30$ $\mathrm{Kg} / \mathrm{m}^{2}$ ), perímetro abdominal (PA) (mujeres normal: $<$ $90 \mathrm{~cm}$., elevado: $\geq 90 \mathrm{~cm}$. y varones normal: $<94 \mathrm{~cm}$, elevado: $\geq 94 \mathrm{~cm}$.), HbA1c (normal: $<7 \%$, elevada: $\geq 7 \%$ ), glucemia en ayunas (normal: $80-130 \mathrm{mg} / \mathrm{dl}$, elevada: > $130 \mathrm{mg} / \mathrm{dl}$ ), presión arterial (normal: <

Tabla 1. Estilo de vida que practican los pacientes con diabetes mellitus tipo 2 .

\begin{tabular}{|c|c|c|c|c|c|c|}
\hline \multirow{3}{*}{ Estilo de vida } & \multicolumn{4}{|c|}{ Sexo } & \multirow{2}{*}{\multicolumn{2}{|c|}{ Total }} \\
\hline & \multicolumn{2}{|c|}{$\mathbf{F}$} & \multicolumn{2}{|c|}{ M } & & \\
\hline & n & $\%$ & $\mathbf{n}$ & $\%$ & n (102) & $\%$ \\
\hline Favorable ( $>80$ pts) & 14 & 30,4 & 12 & 21,4 & 26 & 25,5 \\
\hline Poco favorable (60 - $80 \mathrm{pts})$ & 6 & 13,1 & 24 & 42,9 & 30 & 29,4 \\
\hline Desfavorable $(<60 \mathrm{pts})$ & 26 & 56,5 & 20 & 35,7 & 46 & 45,1 \\
\hline
\end{tabular}


Tabla 2. Variables clínicas del control metabólico en pacientes con diabetes mellitus tipo 2.

\begin{tabular}{|c|c|c|c|c|c|c|}
\hline \multirow{3}{*}{ Variables clínicas } & \multicolumn{4}{|c|}{ Sexo } & \multirow{2}{*}{\multicolumn{2}{|c|}{ Total }} \\
\hline & \multicolumn{2}{|c|}{$\mathbf{F}$} & \multicolumn{2}{|c|}{$\mathbf{M}$} & & \\
\hline & $\mathbf{n}$ & $\%$ & $\mathbf{n}$ & $\%$ & n (102) & $\%$ \\
\hline \multicolumn{7}{|l|}{ IMC } \\
\hline Normal $\left(\mathrm{Kg} / \mathrm{m}^{2}\right)$ & 14 & 30,4 & 23 & 41,1 & 37 & 36,3 \\
\hline Sobrepeso $\left(\mathrm{Kg} / \mathrm{m}^{2}\right)$ & 21 & 45,7 & 23 & 41,1 & 44 & 43,1 \\
\hline Obesidad $\left(\mathrm{Kg} / \mathrm{m}^{2}\right)$ & 11 & 23,9 & 10 & 17,8 & 21 & 20,6 \\
\hline \multicolumn{7}{|l|}{ Perímetro abdominal } \\
\hline Normal $(<90 \mathrm{~cm} . ;<94 \mathrm{~cm})$. & 19 & 41,3 & 28 & 50,0 & 47 & 46,1 \\
\hline Alterado ( $\geq 90 \mathrm{~cm} . ; \geq 94 \mathrm{~cm})$. & 27 & 58,7 & 28 & 50,0 & 55 & 53,9 \\
\hline \multicolumn{7}{|l|}{ Presión arterial } \\
\hline$<140 / 90 \mathrm{~mm} \mathrm{Hg}$ & 25 & 54,3 & 41 & 73,2 & 66 & 64,7 \\
\hline$\geq 140 / 90 \mathrm{~mm} \mathrm{Hg}$ & 21 & 45,7 & 15 & 26,8 & 36 & 35,3 \\
\hline \multicolumn{7}{|l|}{ Glucemia en ayunas } \\
\hline $80-130 \mathrm{mg} / \mathrm{dl}$ & 21 & 45,7 & 17 & 30,4 & 38 & 37,3 \\
\hline$>130 \mathrm{mg} / \mathrm{dl}$ & 25 & 54,3 & 39 & 69,6 & 64 & 62,7 \\
\hline \multicolumn{7}{|l|}{$\mathrm{HbAlc}$} \\
\hline Alterado ( $\geq 7,0 \%)$ & 2 & 4,3 & 2 & 3,6 & 4 & 4,0 \\
\hline Sin datos & 44 & 95,7 & 54 & 96,4 & 98 & 96,0 \\
\hline \multicolumn{7}{|l|}{$\mathrm{C}-\mathrm{LDL}$} \\
\hline Normal (<100 mg/dl) & 10 & 21,7 & 11 & 19,6 & 21 & 20,6 \\
\hline Alterado $(\geq 100 \mathrm{mg} / \mathrm{dl})$ & 36 & 78,3 & 45 & 80,4 & 81 & 79,4 \\
\hline \multicolumn{7}{|l|}{$\mathrm{C}-\mathrm{HDL}$} \\
\hline Alterado $(\leq 50 ; \leq 40 \mathrm{mg} / \mathrm{dl})$ & 36 & 78,2 & 25 & 44,6 & 61 & 59,8 \\
\hline Normal $(>50 ;>40$ mg/dl) & 10 & 21,8 & 31 & 55,4 & 41 & 40,2 \\
\hline \multicolumn{7}{|l|}{ TGC } \\
\hline Normal (<150 mg/dl) & 20 & 43,5 & 19 & 33,9 & 39 & 38,2 \\
\hline Alterado $(\geq 150 \mathrm{mg} / \mathrm{dl})$ & 26 & 56,5 & 37 & 66,1 & 63 & 61,8 \\
\hline
\end{tabular}

140/90 mm Hg, elevada: $\geq 140 / 90 \mathrm{~mm} \mathrm{Hg}$ ), colesterol (C-LDL normal: $<100 \mathrm{mg} / \mathrm{dl}$, elevado: $\geq 100 \mathrm{mg} / \mathrm{dl}$; C-HDL normal: $>50 \mathrm{mg} / \mathrm{dl}$ en mujeres y $>40 \mathrm{mg} /$ $\mathrm{dl}$ en varones) y TGC (normal: $<150 \mathrm{mg} / \mathrm{dl}$, elevado: $\geq 150 \mathrm{mg} / \mathrm{dl})(8,9)$. Estos datos se recolectaron de la historia clínica.

\section{Procedimiento de recolección de datos}

El encargado del Servicio de Programas del Centro Médico EsSalud de Chota proporcionó la información de los usuarios con DM2, previa autorización. Luego de la identificación de los usuarios, se revisaron las historias clínicas completas para recolectar información sobre las variables clínicas y bioquímicas, luego se les visitó en su domicilio para aplicar el cuestionario de estilo de vida, previa aceptación mediante la firma del consentimiento informado.

Se utilizó el Instrumento de Medición de Estilo de Vida en pacientes con DM2 (IMEVID) validado por López et al. (7), el cual tuvo una fiabilidad alfa de Cronbach de 0,81 , coeficiente de correlación test retest de 0,84 , y coeficientes de correlación intraclase para la validez lógica y de contenido fueron de 0,91 y 0,95 , respectivamente. La validez lógica y de contenido 
Tabla 3. Control metabólico en pacientes con diabetes mellitus tipo 2.

\begin{tabular}{|c|c|c|c|c|c|c|}
\hline \multirow{3}{*}{ Control metabólico } & \multicolumn{4}{|c|}{ Sexo } & \multirow{2}{*}{\multicolumn{2}{|c|}{ Total }} \\
\hline & \multicolumn{2}{|c|}{$\mathbf{F}$} & \multicolumn{2}{|c|}{ M } & & \\
\hline & n & $\%$ & $\mathbf{n}$ & $\%$ & n (102) & $\%$ \\
\hline Buen control & 15 & 32,6 & 24 & 42,9 & 39 & 38,2 \\
\hline Mal control & 31 & 67,4 & 32 & 57,1 & 63 & 61,8 \\
\hline
\end{tabular}

Tabla 4. Relación entre estilo de vida y control metabólico en pacientes con diabetes mellitus tipo 2 .

\begin{tabular}{lccccc}
\hline \multirow{2}{*}{ Estilo de vida } & \multicolumn{4}{c}{ Control metabólico } & \\
\cline { 2 - 5 } & \multicolumn{3}{c}{ Buen control } & \multicolumn{3}{c}{ Mal control } & p-valor \\
\cline { 2 - 5 } & $\mathbf{n}$ & $\mathbf{\%}$ & $\mathbf{n}$ & $\mathbf{\%}$ & \\
\hline Favorable $(>80 \mathrm{pts})$ & 12 & 30,8 & 14 & 22,2 & $0,000^{*}$ \\
Poco favorable $(60-80 \mathrm{pts})$ & 25 & 64,1 & 5 & 7,9 & $0,000^{* *}$ \\
Desfavorable $(<60 \mathrm{pts})$ & 2 & 5,1 & 44 & 69,9 & \\
\hline${ }^{*}$ Chi cuadrado $(\mathrm{p}<0,05) .{ }^{* *}$ Coeficiente & & &
\end{tabular}

fue evaluada por un panel multidisciplinario de 16 profesionales de la salud conformado por médicos internistas, endocrinólogos, psicólogos clínicos, médicos familiares, nutricionistas e investigadores clínicos, con más de cinco años de experiencia en la atención de pacientes con diabetes.

Para adaptar el instrumento IMEVID al contexto y obtener una validez de respuesta, se realizó una prueba piloto con 18 pacientes que presentaban DM2 con edades de 30 a 60 años, atendidos en establecimientos de salud del Ministerio de Salud de la ciudad de Chota. Se obtuvo una fiabilidad del instrumento de 0,89 mediante la prueba estadística KR-20. La guía de interpretación diagnóstica para el control metabólico, fue adaptada de la ADA (8).

\section{Análisis estadístico}

Se determinaron frecuencias absolutas y relativas para las variables categóricas y se categorizaron las variables numéricas según el propósito del estudio. Para el análisis relacional se utilizó el Chi Cuadrado de Independencia y el Coeficiente Gamma, se consideró estadísticamente significativo un $\mathrm{p}<0,05$. El análisis estadístico fue realizado en el programa estadístico SPSS versión 22.

\section{Aspectos éticos}

Los participantes asintieron firmando el consentimiento informado, previa información de los objetivos y características del estudio. El manejo de los datos fue discreto manteniendo la confidencialidad $\mathrm{y}$ una conducta responsable en investigación en todo momento.

\section{RESULTADOS}

Se trabajó con 102 pacientes con DM2. El estilo de vida fue desfavorable en el $45,1 \%$ de participantes (tabla 1). El 43,1\% presentaron sobrepeso, el 53,9\% tenían PA elevado, el 64,7\% presión arterial normal, $62,7 \%$ glucemia en ayunas elevada, $96,0 \%$ no contaron con examen de HbA1c, 79,4\% C-LDL elevado, 59,8\% tenían valores disminuidos de C-HDL y $61,8 \%$ tenían TGC elevados (tabla 2).

Referente al control metabólico de la DM2, el 61,8\% presentó mal control metabólico (tabla 3). Se encontró una relación directa y altamente significativa entre el estilo de vida desfavorable y el mal control metabólico en pacientes con DM2 $(p=0,000)($ tabla 4$)$.

\section{DISCUSIÓN}

El objetivo del estudio fue determinar la relación entre el estilo de vida y el control metabólico en pacientes con DM2 de Chota, Cajamarca; se encontró una relación directa y altamente significativa entre el estilo de vida desfavorable y el mal control metabólico de la enfermedad.

El distrito de Chota se ubica en la parte norte central del Perú, en el centro del departamento de Cajamarca. $\mathrm{Su}$ capital se encuentra en la meseta de Acunta a 2388 
msnm y a $150 \mathrm{~km}$ al norte de Cajamarca o a $219 \mathrm{~km}$ al este de Chiclayo, Lambayeque. Su población figura como uno de los grupos robustos más pobres a nivel nacional, con ciertas limitaciones de acceso a servicios básicos de saneamiento y de salud (10). El Centro Médico de EsSalud de Chota pertenece a la Red Asistencial Lambayeque, solamente brinda servicios de salud a los asegurados, los cuales se limitan a las prestaciones básicas de prevención, promoción, recuperación y rehabilitación de la salud (11).

La mayor proporción de participantes presentó un estilo de vida desfavorable y mal control metabólico de la DM2, manifestado por sobrepeso, PA alterado, hiperglucemia, C-LDL y TGC elevados y C-HDL disminuido; debido probablemente a prácticas inadecuadas de salud.

El estilo de vida fue desfavorable en casi la mitad de los pacientes evaluados. Dos estudios mexicanos también encontraron estilo de vida desfavorable en $62,2 \%$ y $76,6 \%(12,13)$, por tratarse de un país muy industrializado. En el estudio, más del $60 \%$ de la población estudiada tenía sobrepeso u obesidad. En poblaciones mexicanas estudiadas se ha encontrado sobrepeso en $47 \%$ y obesidad en $40 \%(14,12)$. Las prácticas inadecuadas de los pacientes se explican por el acelerado ritmo de vida de la sociedad actual, y se presenta por el consumo frecuente de productos industrializados y comidas rápidas, inactividad física, consumo de alcohol y tabaco y adherencia insuficiente al tratamiento $(15,16)$.

El PA alterado es un indicador de suma importancia en los pacientes con DM2. Un estudio realizado en Trujillo, Perú, encontró resultados similares; es decir, PA con riesgo muy elevado para mujeres $(62 \%)$ y varones $(47 \%)(17)$, por dedicarse a labores administrativas principalmente. Los individuos con obesidad abdominal, aunque muestren un peso saludable, tienen mayor riesgo de presentar cifras elevadas de glicemia, presión arterial o colesterolemia que aquellas personas con IMC de sobrepeso, pero cuyo exceso de grasa es periférico (18).

Estudios realizados en México, Ecuador y Perú también encontraron hiperglicemia en los usuarios $(12,14,19,20,21)$, siendo común para los diferentes contextos. La glucemia en ayunas en un paciente con DM2 puede elevarse por diferentes razones, como: ingestión de demasiada comida (una comida con más carbohidratos de lo usual), no tener una vida activa, insuficiente insulina o medicamentos (por vía oral) para la diabetes, efectos secundarios de otros medicamentos (por ejemplo, esteroides, medicamentos antipsicóticos), estrés (el estrés aumenta la producción de hormonas como cortisol, que suben el nivel de glucosa en sangre) o deshidratación (22).

La monitorización de la glucemia debe constituir un aspecto fundamental en el control y cuidado de los pacientes con DM2. Se ha demostrado que si se retrasa la exposición a la hiperglucemia de manera precoz se puede retrasar las complicaciones vasculares (23).

Solamente cuatro pacientes tenían valoración de HbA1c, los que a su vez presentaban un valor elevado, debido a que el Centro Médico EsSalud de Chota no cuenta con el método de HPLC u otros afines para su determinación. Dos estudios reportan resultados similares al nuestro, con valores de $\mathrm{HbA} 1 \mathrm{c}$ promedio de 7,4\% y $13,32 \%(14,24)$. El aumento de la HbA1c es proporcional al aumento de las complicaciones de la patología asociada del paciente (25).

Los resultados del C-LDL y C-HDL guardan relación con estudios de Ecuador y España, que también encontraron cifras elevadas para C-LDL y cifras disminuidas para C-HDL $(20,26)$. La hipercolesterolemia promueve la aterosclerosis, por ende representa un importante factor de riesgo cardiovascular en los pacientes con DM2 (27). Las dislipidemias tienen un efecto pronóstico diferente de acuerdo a la edad de los individuos (en el estudio más de la mitad eran menores de 60 años). Mientras más joven sea la persona cuando se presenta la dislipidemia, mayor es el impacto sobre la esperanza de vida, dependiendo en gran parte de la identificación de factores de riesgo y la concienciación acerca de los efectos del consumo alimentario no saludable (28).

Los bajos niveles de C-HDL pueden deberse a la inactividad física, el sobrepeso, la obesidad y el hábito de fumar, lo cual conlleva a un mayor riesgo de enfermedades cardiovasculares (29). El desarrollo de nuevos fármacos que modifiquen en forma más significativa los niveles de C-HDL para su uso combinado con las estatinas permite vislumbrar una estrategia más integral para el tratamiento de la dislipidemia en el paciente con DM2 (30).

Dos estudios muestran cifras parecidas a las nuestras, con valores $>150 \mathrm{mg} / \mathrm{dl}$ de TGC en el $66,7 \%$ de usuarios y valor promedio de $200,25 \mathrm{mg} /$ dl $(12,14)$. Las causas más comunes de un nivel alto de TGC son la obesidad, sedentarismo y la DM2 mal 
controlada; especialmente si existe consumo excesivo de carbohidratos o alcohol (31).

Más de la mitad de pacientes presentaron mal control metabólico, de forma similar a lo reportado por un estudio español (26). Esta situación es el resultado de las alteraciones de los distintos indicadores descritos. Si el mal control metabólico persiste a lo largo del tiempo, será el responsable de la aparición de complicaciones y daño a nivel de diferentes órganos y sistemas (32).

Se encontró una relación directa y altamente significativa entre el estilo de vida y el control metabólico en pacientes con DM2. Algunos estudios reportan resultados parecidos al nuestro; es decir, presentan una relación significativa entre el estilo de vida y el control metabólico (12) y estilo de vida favorable con un menor valor de HbAlc (13), aportando de esta manera evidencia a favor de la hipótesis planteada.

Las principales limitaciones del estudio fueron el uso de fuentes secundarias para obtener los valores de las variables clínicas de los pacientes y la población estudiada del Centro Médico EsSalud no es representativa de toda la ciudad de Chota.

En conclusión, gran proporción de los pacientes practicaban un estilo de vida desfavorable y tenían mal control metabólico. Existe una relación directa y altamente significativa entre el estilo de vida y el control metabólico de los pacientes, el estilo de vida desfavorable se asoció a mal control metabólico de la enfermedad.

Se recomienda al Centro Médico, la implementación del examen de $\mathrm{HbAlc}$ en los pacientes, por ser uno de los indicadores más específicos para evaluar el control metabólico de la DM2 y la ejecución de planes terapéuticos y educativos que mejoren el estilo de vida de los pacientes.

\section{Correspondencia:}

José Ander Asenjo-Alarcón

Correo electrónico: ander1213@hotmail.com

\section{REFERENCIAS BIBLIOGRÁFICAS}

1. Gómez PR. Cambio en el estilo de vida en Diabetes tipo 2: una leyenda urbana. Unidad de Endocrinología, Instituto Autónomo Hospital
Universitario de Los Andes Universidad de Los Andes, Mérida, Venezuela. Rev Venez Endocrinol Metab. 2013; 11(2):56-8.

2. Reyes CM, López AL, Flores PL. Invalidez laboral por diabetes mellitus tipo 2: experiencia en 393 pacientes de ciudad Juárez, México. Arch Med Fam. 2010; 12(4):110-5.

3. Seclen SN, Rosas ME, Arias AJ, et al. Prevalence of diabetes and impaired fasting glucose in Peru: report from PERUDIAB, a national urban population based longitudinal study. BMJ Open Diabetes Research and Care. 2015; 3:e000110. doi:10.1136/bmjdrc-2015000110S

4. Organización Mundial de la Salud. Obesidad y Sobrepeso. Ginebra, Suiza: Organización Mundial de la Salud; 2015.

5. Álvarez DD, Sánchez AJ, Gómez GG, Tarqui MC. Sobrepeso y obesidad: Prevalencia y determinantes sociales del exceso de peso en la población peruana (2009-2010). Rev Perú Med Exp Salud Pública. 2012; 29(3):303-13. doi: http://dx.doi. org/10.17843/rpmesp.2012.293.362

6. Organización Mundial de la Salud. 10 datos sobre la diabetes. Ginebra, Suiza: Organización Mundial de la Salud; 2014.

7. López CJ, Rodríguez MJ, Ariza AC, Munguía MC. Construcción y validación inicial de un instrumento para medir el estilo de vida en pacientes con diabetes mellitus tipo 2. Salud Publica Mex. 2003; 45(4):259-68.

8. American Diabetes Association. Standards of medical care in diabetes - 2015. Diabetes Care. 2015; 38(Suppl 1):S1-S94.

9. Seclén S, Villena J, Larrabure G, Manrique H, Salazar CA, Zapata L, Calderón J. Consenso peruano sobre prevención y tratamiento de la diabetes mellitus tipo 2, síndrome metabólico y diabetes gestacional. Congreso Internacional en prediabetes y síndrome metabólico. Lima, Perú: Sociedad Peruana de Endocrinología; 2012. Disponible en: https://www. endocrinoperu.org/sites/default/files/Consenso $\% 20$ Peruano\%20sobre $\% 20$ Prevencion $\% 20$ y $\% 20$ Tratamiento\%20de\%20Diabetes\%20Mellitus\%20 $2 \% 20$ Sindrome $\% 20$ Metabolico $\% 20 \mathrm{y} \% 20$ Diabetes $\%$ 20Gestacional.pdf

10. Instituto Nacional de Estadística e Informática. Mapa de pobreza monetaria provincial y distrital 2018. Lima, Perú: Instituto Nacional de Estadística e Informática; 2020.

11. EsSalud. Reglamento de organización y funciones del Seguro Social de Salud. Lima, Perú: EsSalud, Gerencia Central de Planeamiento y Desarrollo; 2016.

12. Gómez-Aguilar PI, Avila-Sansores GM, CandilaCelis JA. Estilo de vida y control metabólico en personas con diabetes tipo 2, Yucatán, México. 
Rev Enferm Inst Mex Seguro Soc. 2012; 20(3):12329.

13. Urbán-Reyes BR, Coghlan-López JJ, CastañedaSánchez O. Estilo de vida y control glucémico en pacientes con Diabetes Mellitus en el primer nivel de atención. Aten Fam. 2015; 22(3):68-71.

14. Figueroa-Suárez ME, Cruz-Toledo JE, OrtizAguirre AR, Lagunes-Espinosa AL, Jiménez-Luna J, Rodríguez-Moctezuma JR. Estilo de vida y control metabólico en diabéticos del programa DiabetIMSS. Gac Med Mex. 2014; 150:29-34.

15. Ramírez VR, Ojeda ML, Tordecilla MA, Peña JC, Meneses JF. El consumo regular de bebidas azucaradas incrementa el perfil lipídico-metabólico y los niveles de adiposidad en universitarios de Colombia. Rev Colomb Cardiol. 2015; 22(4):157204.

16. Gómez HR. Hacia un manejo integral del paciente con diabetes y obesidad. Posicionamiento de la SEMI, SED, redGDPS, SEC, SEEDO, SEEN, SEMERGEN y SEMFYC. Rev Clinica Española. 2015; 215(6):301-62.

17. Ponce PK, Benites PK. Factores de riesgo de diabetes mellitus tipo 2 en el personal administrativo de la Universidad Privada Antenor Orrego. Trujillo, 2014. Tesis Licenciatura. Trujillo, Perú: Universidad Privada Antenor Orrego; 2015. 119 pp.

18. Darias-Garzón R. Obesidad abdominal factor de riesgo para diabetes tipo 2. Ciudad de México: Federación Mexicana de Diabetes; 2017.

19. Pilamala JD. Actividad física en pacientes con diabetes mellitus tipo 2 que asisten al club de diabéticos del hospital básico Píllaro. Tesis Licenciatura. Ecuador: Universidad Técnica de Ambato; 2015. 114 pp.

20. Beltrán LE. Estado metabólico y factores asociados en los pacientes diabéticos tipo 2 que asisten a consulta externa del hospital de Girón. 2014. Tesis de Especialidad en Medicina Interna. Ecuador: Universidad de Cuenca; 2015. 52 pp.

21. Alegría GK, Manrique JE, Pérez MR. Características sociodemográficas - clínicas y estilo de vida en los pacientes con diabetes mellitus tipo 2, del C.S. San Juan, Iquitos - 2014. Tesis Licenciatura. Amazonas, Perú: Universidad Nacional de la Amazonía Peruana; 2014. 93 pp.

22. Asociación Americana de Diabetes. Factores que afectan el nivel de glucosa en la sangre. Boletín electrónico. Alto a la Diabetes. Washington DC, Estados Unidos: Asociación Americana de Diabetes; 2015.
23. De la Hera GJ, García RJ, Delgado AE, Ferreiro J. Tiempo de evolución de la diabetes mellitus como factor determinante en la modificación de la reactividad plaquetaria. Rev Esp Cardiol. 2014; 67(3):241-44. doi: 10.1016/j.recesp.2013.11.004

24. Alarcón NS, Alonso MM, Cadena F, Rodriguez Y. Estilo de vida y control glucémico en pacientes con Diabetes Mellitus tipo 2 de una Institución de Seguridad Social de Nuevo Laredo, Tamaulipas. Rev Enferm Universitaria. 2007;4(2):5-10.

25. Francisco BB, Nunes SJ, Ferraz C, Namo CA, Sargaço R. Aplicabilidade clínica da hemoglobina glicada na evolução do paciente com hiperglicemia hospitalar. Rev Soc Bras Clínica Médica. 2015;13(2):111-3.

26. Pesqueira FP, Grandes IJ, Rodríguez GC, Molinos CS, González VL, De la Cruz AJ, et al. Grado de control metabólico en pacientes ambulatorios con diabetes mellitus tipo 2 en Medicina Interna. Estudio BARVI. Gac Médica Bilbao. 2012; 109(2):52-8.

27. Cofan PM. Mecanismos básicos. Absorción y excreción de colesterol y otros esteroles. Clínica e Investig en Arterioscler. 2014; 26(1):41-7.

28. Colcha VN. Prevalencia de sobrepeso, obesidad, dislipidemias en el personal activo de la brigada de caballería blindada $\mathrm{N}^{\circ} 11$ Galápagos Riobamba 2010. Riobamba, Ecuador: Escuela Superior Politécnica de Chimborazo; 2011.

29. Saá GV. Prevalencia de dislipidemias con relación a sobrepeso y obesidad en los servidores activos de la ESPOCH. Año 2013. Tesis Licenciatura. Riobamba, Ecuador: Escuela Superior Politécnica de Chimborazo; 2014. 91 pp.

30. Rigotti RA. Actualización en el manejo del colesterol HDL bajo. Rev Médica Clínica Las Condes. 2012; 23(6):689-92.

31. Miller M, Stone N, Ballantyne C, et al. Triglycerides and cardiovascular disease: A scientific statement from the American Heart Association. Circulation. 2011; 123(20):2292-333.

32. De La Haza MC. Contribución al estudio de los factores relacionados con la microangiopatía diabética. Tesis Doctoral. Madrid, España: Universidad Complutense de Madrid; 2013. 109 pp.

Recibido: 30/03/2019

Aceptado: 20/01/2020 